ISSN 1112-9867

http://www.jfas.info

\title{
GENERALIZED PROJECTIVE SERIES SYNCHRONIZATION BETWEEN CHAOTIC SYSTEMS AND ITS APPLICATION
}

\author{
N. A. A. Fataf ${ }^{1,2,}{ }^{*}$ and M. R. M. Said ${ }^{2}$ \\ ${ }^{1}$ Centre for Defence Foundation Studies, UniversitiPertahananNasional Malaysia, 57000 \\ Kuala Lumpur, Malaysia \\ ${ }^{2}$ Institute Mathematical Research, Universiti Putra Malaysia, 43400 Serdang, Selangor, \\ Malaysia
}

Published online: 10 September 2017

\begin{abstract}
The existence of generalized projective series synchronization scheme between different chaotic systems is investigated. The synchronization scheme consists of three 3D systems and one 4D system to show the generalized projective series synchronization. The synchronization can be well implemented with non-linear adaptive coupling. Sufficient conditions for synchronization are also derived according to Lyapunov stabilization theory. Numerical simulations show the effectiveness of the analytical result. The application of generalized projective series synchronization scheme is implemented to image encryption. The correlation between adjacent pixels in original image and encrypted image is analyzed to show the effectiveness of the synchronization scheme.
\end{abstract}

Keywords: generalized projective; synchronization; image encryption.

Author Correspondence, e-mail: n.aisyah@upnm.edu.my

doi: http://dx.doi.org/10.4314/jfas.v9i3s.24 


\section{INTRODUCTION}

Synchronization occurs when a drive chaotic system transmits one or more of its driving signal to the response system which can be either identical or non-identical to the drive system. If the drive system does not synchronize with the response system, it is possible to design a controller at the response system which enforces synchronization to occur. Once the drive-response system synchronizes, it is possible to design a communication model based on the principles of chaotic synchronization. Since chaotic dynamical system is a deterministic system, its-random behaviour can be very helpful. A small perturbation eventually causes a large change in the state of system. Chaotic synchronization communication scheme is the scheme that transmit a signal carried from the transmitter to the receiver by a chaotic signal through an analog channel [1]

Synchronization attracted with much attention in many fields such as in biological [2], chemical [3], physical [5] and social systems [5]. Synchronization and similar concepts also abound in technical sciences and engineering, including computer science [6], control theory [7] and communications [8]. Over the past decades, several new types of synchronization have been investigated such as generalized synchronization [9], phase synchronization [10], lag synchronization [11], etc. Many works have been done for generalized synchronization where there exists an essential difference between the coupled systems [12]. Generalized projective synchronization of chaotic systems can synchronize up to a constant scaling matrix [13-14]. Some examples of generalized projective synchronization are hybrid synchronization [15], complete synchronization [16], projective synchronization [17], fuzzy generalized projective synchronization [18] and anti-synchronization [19]. Secure communication [20-21] and secure data encryption [22-24] are the examples of the applications of the generalized projective synchronization in the field of communication.

A single pair of one way coupled oscillations cannot be applied for multiuser communication systems. Multiplexing technique is very important issue for high capacity communications [25]. Dual synchronization of chaotic maps has been investigated by [26]. Dual synchronization is coupled synchronization of two drive systems with two response systems where the systems can be either identical or non-identical. Dual synchronization of the Lorenz 
and Rossler systems is studied by [27], which the output signal from the drive systems is a scalar signal. Here, extended of a series synchronization by using various drive and response chaotic systems where the systems can be identical or non-identical is proposed. Recently, a novel scheme of dual combination synchronization is investigated for six chaotic systems using adaptive control [28]. The theoretical and the numerical simulations in this research proof that the effectiveness and correctness of the dual combination synchronization. In [29] also investigated the existence of the dual synchronization behaviour between a pair of chaotic and hyperchaotic systems via nonlinear controller.

The generalized projective synchronization between identical and non-identical ordinary differential equation systems is studied. We consider a series of different order chaotic and hyperchaotic systems. The synchronization can be well implemented with nonlinear coupling, which can be derived using Lyapunov stability theory. Then, the synchronization implemented with the image encryption. The correlation of two adjacent pixels' analysis shows that the synchronization is well implemented to the image encryption. Consider the systems [30] as follows:

$$
\begin{gathered}
\dot{x_{1}}=y_{1} z_{1} \\
\dot{y}_{1}=x_{1}-y_{1}(1) \\
\dot{z}_{1}=a-x_{1} y_{1} \\
\dot{x_{2}}=y_{2} z_{2} \\
\dot{y}_{2}=x_{2}-y_{2}(2) \\
\dot{z}_{2}=a-x_{2} y_{2} \\
\dot{x_{3}}=y_{3} z_{3} \\
\dot{y}_{3}=x_{3}-y_{3}(3) \\
\dot{z}_{3}=a-x_{3} y_{3}
\end{gathered}
$$

where $x_{1}, y_{1}, z_{1}$ as the state variables and $a$ as the parameter for the system (1), $x_{2}, y_{2}, z_{2}$ as the state variables and $b$ as the parameter for the system (2) and $x_{3}, y_{3}, z_{3}$ as the state variables and $c$ as the parameter for the system (3). We also consider the Lorenz-stenflo system [31-32] as follows:

$$
\begin{gathered}
\dot{x}_{4}=\alpha\left(y_{4}-x_{4}\right)+\gamma w_{4} \\
\dot{y}_{4}=x_{4}\left(r-z_{4}\right)-y_{4}(4) \\
\dot{z}_{4}=x_{4} y_{4}-\sigma z_{4} \\
\dot{w}_{4}=-x_{4}-\alpha w_{4}
\end{gathered}
$$

where $x_{4}, y_{4}, z_{4}, w_{4}$ are the state variables and $\alpha, \gamma, r, \sigma$ as the parameters. Fig. 1 shows the 
chaotic attractors for system (1)-(4) respectively. The system (1) is chaotic for $a=1$, while the system (2) is chaotic when $b=0.5$. When $b=0.3$ we can see the chaotic attractor for the system (3) and hyperchaotic attractor when $\alpha=1, \gamma=1.5, r=26, \sigma=0.7$ for the system (4).

System 1

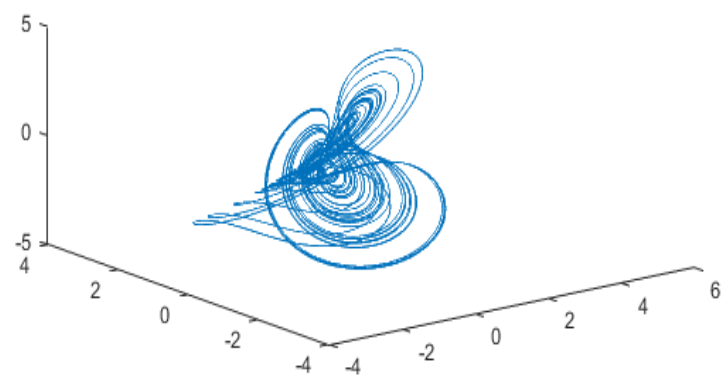

System 3

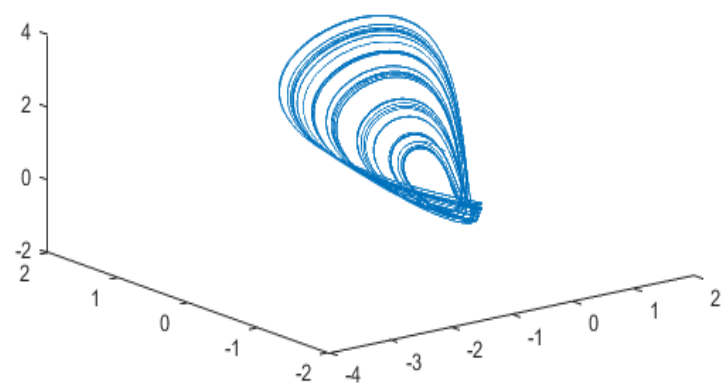

System 2

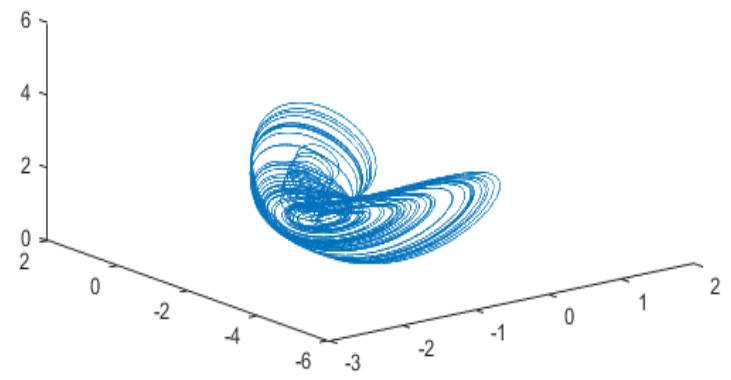

System 4

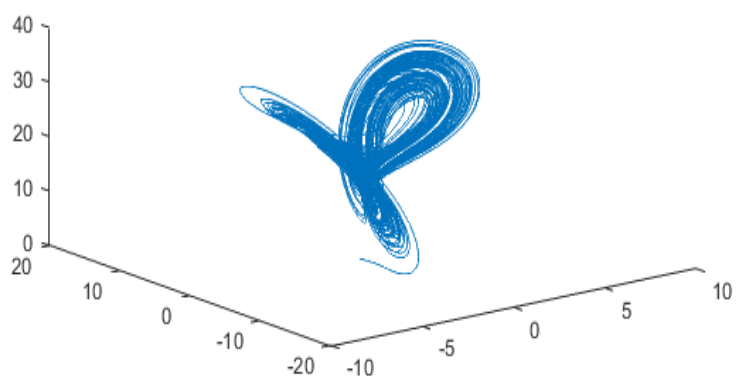

Fig.1. Phase space diagrams of the chaotic attractor for the system (1)-(4)

\section{METHODOLOGY}

In this section, the asymmetric synchronization behavior between a series of chaotic systems is studied. The scheme is illustrated in the Fig. 2. 


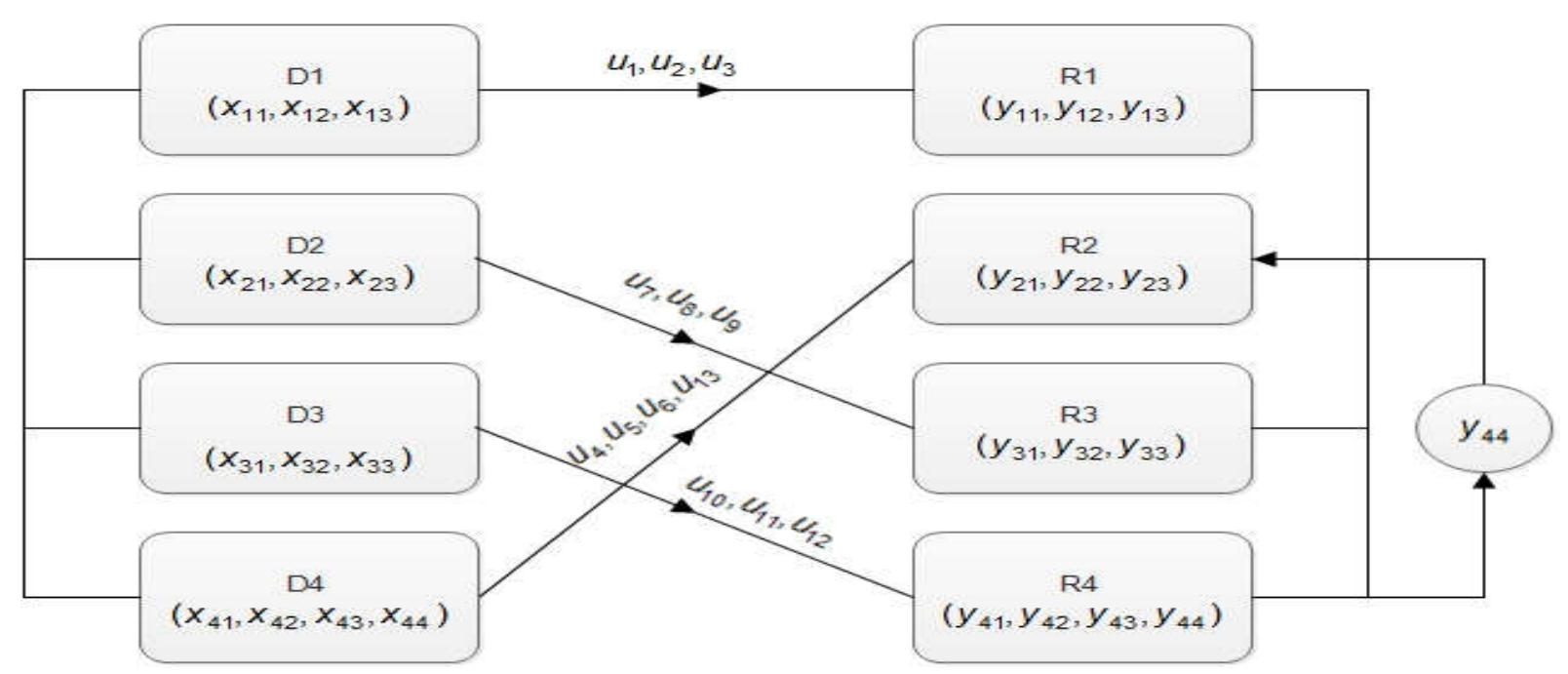

Fig.2.Asymmetric synchronization scheme for a series of systems

From Fig. 2, we can see that the driving and response systems are not identical. D2 is coupled with R3 using controller $u_{7}, u_{8}, u_{9}$. Similarly, D4 with R2 by the controller $u_{4}, u_{5}, u_{6}, u_{13}$. We consider the drive systems as:

$$
\begin{gathered}
D_{1}:\left(\dot{x}_{11}, \dot{x}_{12}, \dot{x}_{13}\right)=\left(x_{12} x_{13}, x_{11}-x_{12}, a-x_{11} x_{12}\right) \\
D_{2}:\left(\dot{x}_{21} \dot{x}_{22} \dot{x}_{23}\right)=\left(x_{22}+x_{23}-x_{21}+b_{22} x_{2}{ }_{1}-x_{23}\right) \\
D_{3}:\left(\dot{x}_{31}, \dot{x}_{32}, \dot{x}_{33}\right)=\left(x_{31} x_{32}-x_{33}, x_{31}-x_{32}, x_{31}+x_{31}+a x_{33}\right) \\
D_{4}:\left(\dot{x}_{41}, \dot{x}_{42}, \dot{x}_{43}, \dot{x}_{44}\right)=\left(\alpha\left(x_{42}-x_{41}\right)+\gamma x_{44}, x_{41}\left(r-x_{43}\right)-x_{42}, x_{41} x_{42},-x_{41}-\alpha x_{44}\right)
\end{gathered}
$$

and the same set with different combinations for the response as follows:

$$
\begin{gathered}
R_{1}:\left(\dot{y}_{11}, \dot{y}_{12}, \dot{y}_{13}\right)=\left(y_{12} y_{13}+u_{1},+y_{11}-y_{12}, a-y_{11} y_{12}+u_{3}\right) \\
R_{2}:\left(\dot{y}_{21} \dot{y}_{22} \dot{y}_{23}\right)=\left(y_{22}+y_{23}+u_{4},-y_{21}+b y_{2}+u_{5}, y_{2}{ }_{1}-y_{23}+u_{6}\right) \\
R_{3}:\left(\dot{y}_{31}, \dot{y}_{32}, \dot{y}_{33}\right)=\left(y_{31}+y_{32}-y_{33}+u_{9}, y_{31}-y_{32}+u_{8}, y_{31}+c y_{33}+u_{9}\right) \\
R_{4}:\left(\dot{y}_{41}, \dot{y}_{42}, \dot{y}_{43}, \dot{y}_{44}\right)=\left(\begin{array}{c}
\alpha\left(y_{42}-y_{41}\right)+\gamma y_{44}+u_{10}, y_{41}\left(r-y_{43}\right)-x_{42}+u_{11}, \\
y_{41} y_{42}+u_{12},-y_{41}-\alpha y_{44}+u_{13}
\end{array}\right)
\end{gathered}
$$

where $u=\left(u_{1}, u_{2}, \ldots, u_{13}\right)^{T}$ is the active control function to be determined. We need to determine the controller $u$ which is required for system (6) to synchronize with system (5). For this purpose, let the error dynamics between (5) and (6) be

$$
\begin{array}{r}
e_{1}=x_{11}-h y_{11}, e_{2}=x_{12}-h y_{12}, e_{3}=x_{13}-h y_{13} \\
e_{4}=x_{21}-h y_{31}, e_{5}=x_{22}-h y_{32}, e_{6}=x_{23}-h y_{33} \\
e_{7}=x_{31}-h y_{41}, e_{8}=x_{32}-h y_{42}, e_{9}=x_{33}-h y_{43}(7) \\
e_{10}=x_{41}-h y_{44}, e_{11}=x_{42}-h y_{21} e_{12}=x_{43}-h y_{22} \\
e_{13}=x_{44}-h y_{23}
\end{array}
$$

Here, $h$ is a desired scaling factor. Thus, the error system can be given by: 


$$
\begin{gathered}
\dot{e}_{1}=x_{12} x_{13}-h\left(y_{12} y_{13}+u_{1}\right) \\
\dot{e}_{2}=x_{11}-x_{12}-h\left(y_{11}-y_{12}+u_{2}\right) \\
\dot{e}_{3}=a-x_{11} x_{12}-h\left(a-y_{11} y_{12}+u_{3}\right) \\
\dot{e}_{4}=x_{22}+x_{23}-h\left(y_{31}-y_{33}+u_{7}\right) \\
\dot{e}_{5}=x_{21}+b x_{2}-h\left(y_{31}-y_{32}+u_{8}\right) \\
\dot{e}_{6}=x_{2}{ }_{1}-x_{23}-h\left(y_{31}+c y_{33}+u_{9}\right) \\
\dot{e}_{7}=x_{31} x_{32}-x_{33}-h\left(\alpha\left(y_{42}-y_{41}\right)+\gamma y_{44}+u_{10}\right)(8) \\
\dot{e}_{8}=x_{31}-x_{32}-h\left(y_{41}\left(r-y_{43}\right)+y_{42}+u_{11}\right) \\
\dot{e}_{9}=x_{31}+c x_{33}-h\left(y_{41} y_{42}-\sigma y_{43}+u_{12}\right) \\
\dot{e}_{10}=\alpha\left(x_{42}-x_{41}\right)+\gamma x_{44}-h\left(-y_{41}-\alpha y_{44}+u_{13}\right) \\
\dot{e}_{11}=x_{41}\left(r-x_{43}\right)+x_{42}-h\left(y_{2}+y_{2}+u_{4}\right) \\
\dot{e}_{12}=x_{41} x_{42}-\alpha x_{43}-h\left(-y_{2}-b y_{2}+u_{5}\right) \\
\dot{e}_{13}=-x_{41}-\alpha x_{44}-h\left(y_{2}{ }_{1}-y_{2}+u_{6}\right)
\end{gathered}
$$

Re-define the active control function $u=\left(u_{1}, u_{2}, \ldots, u_{13}\right)^{T}$ as

$$
\begin{aligned}
& u_{1}=\frac{1}{h}\left(x_{12} x_{13}-\frac{x_{12} x_{13}}{h}+\frac{x_{12} e_{3}}{h}+\frac{e_{2} x_{13}}{h}+\frac{e_{2} e_{3}}{h}+e_{1}\right) \\
& u_{2}=\frac{e_{1}}{h} \\
& u_{3}=\frac{1}{h}\left(a-x_{11} x_{12}-h a-\frac{x_{11} x_{12}}{h}+\frac{x_{12} e_{2}}{h}+\frac{x_{12} e_{1}}{h}+\frac{e_{1} e_{2}}{h}+e_{3}\right) \\
& u_{4}=\frac{1}{h}\left(r x_{41}-x_{41} x_{43}-x_{42}-x_{43}+e_{12}-x_{44}+e_{13}+e_{11}\right) \\
& u_{5}=\frac{1}{h}\left(x_{41} x_{42}-\sigma x_{43}+x_{42}-e_{11}-b x_{43}+2 b e_{12}\right) \\
& u_{6}=\frac{1}{h}\left(-x_{41}-\alpha x_{44}-\frac{x_{42}^{2}}{h}+\frac{2 x_{42} e_{11}}{h}-\frac{e_{11}^{2}}{h}+x_{44}\right) \\
& u_{7}=\frac{1}{h}\left(x_{22}+2 x_{23}-\frac{x_{2} x_{22}}{h}+\frac{x_{2} e_{5}}{h}+\frac{x_{2} e_{4}}{h}+\frac{e_{4} e_{5}}{h}-e_{6}+e_{4}\right) \\
& u_{8}=\frac{1}{h}\left(-2 x_{21}+b x_{22}+x_{22}+e_{4}\right) \\
& u_{9}=\frac{1}{h}\left(x_{2}{ }_{1}^{2}-x_{23}-x_{21}+e_{4}-c x_{23}+2 c e_{6}\right) \\
& u_{10}=\frac{1}{h}\left(x_{31} x_{32}-x_{33}-\alpha x_{32}+\alpha e_{8}+\alpha x_{31}-\gamma x_{41}+\gamma e_{10}\right) \\
& u_{11}=\frac{1}{h}\left(x_{31}-r x_{31}+c x_{33}+\frac{x_{31} x_{32}}{h}-\frac{x_{31} e_{8}}{h}-\frac{x_{32} e_{7}}{h}+\frac{e_{7} e_{9}}{h}\right) \\
& u_{12}=\frac{1}{h}\left(x_{31}+c x_{33}-\frac{x_{31} x_{32}}{h}+\frac{x_{31} e_{8}}{h}+\frac{x_{32} e_{7}}{h}-\frac{e_{7} e_{8}}{h}-\sigma x_{33}\right) \\
& u_{13}=\frac{1}{h}\left(\alpha x_{42}+\gamma x_{44}+x_{31}-e_{7}\right)
\end{aligned}
$$

Substituting (9) in (8) gives 


$$
\begin{gathered}
\dot{e}_{1}=-e_{1}, \dot{e}_{2}=-e_{2}, \dot{e}_{3}=-e_{3}, \dot{e}_{4}=-e_{4}, \dot{e}_{5}=-e_{5}, \\
\dot{e}_{6}=-e_{6}, \dot{e}_{7}=-e_{7}, \dot{e}_{8}=-e_{8}, \dot{e}_{9}=-e_{9}, \dot{e}_{10}=-e_{10},(10) \\
\dot{e}_{11}=-e_{11}, \dot{e}_{12}=-e_{12}, \dot{e}_{13}=-e_{13}
\end{gathered}
$$

For the asymptotic stability of the errors, consider a positive Lyapunov functional in the form

$V=\frac{1}{2} \sum_{i=1}^{13} e_{i}^{2}$

According to Lyapunov stabilization, we need to find the sufficient condition for $e_{i} \rightarrow 0$ as $t \rightarrow \infty$ where $i=1,2, \ldots, 13$ so that its derivative along trajectories of (1)-(4), $\dot{V}<0$. These choices ensures that the error states (7) exponentially converge to zero as time $t \rightarrow \infty$. By Lyapunov stabilization theory, the sufficient condition for generalized projective series synchronization are $b>0, c>0, \alpha>0, \sigma>0$. Therefore, when (10) is stabilized by the feedback control input function $u$, the error will converge to zero as $t \rightarrow \infty$ which implies that the systems (5) and (6) are globally synchronized.

\section{RESULTS AND DISCUSSION}

For the purpose of numerical simulation fix as in Fig. 1 to ensure the chaotic motion. Next, choose the initial condition for driving systems as and the initial condition for the response systems are with the value of $h=2$. 


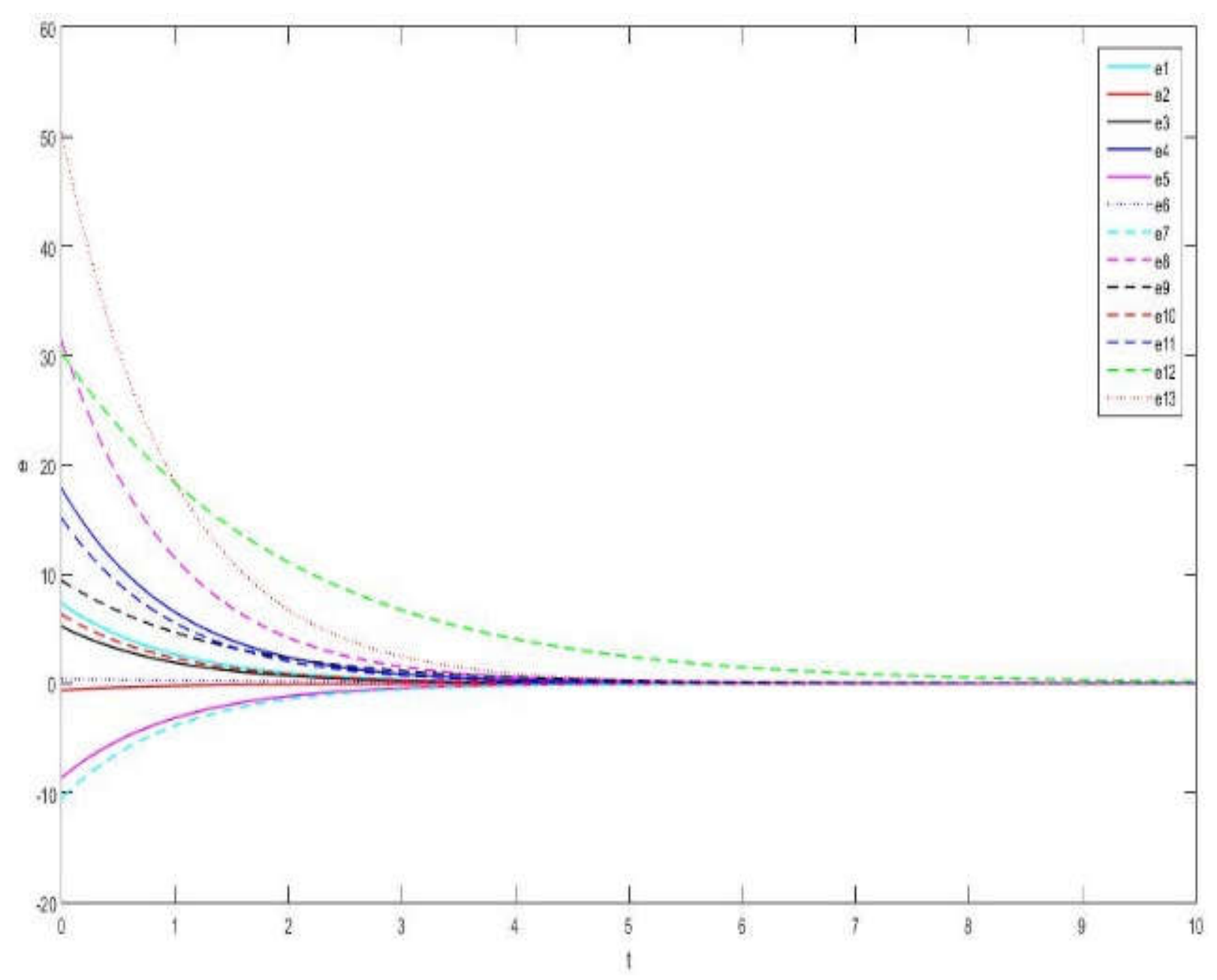

Fig.3. The error dynamics corresponding to generalized projective series synchronization

In Fig. 3, it is obvious that drive-response systems are globally synchronized after the controller is activated. Clearly that the system (6) traces the dynamics of the system (5).

\subsection{Application: Chaos-Based Image Encryption}

Image encryption schemes is one of the field that is most increasingly studied by researchers to meet the demand for real-time secure image transmission over the internet via wireless network. Traditional image encryption such as data encryption standard (DES) and AES are not suitable for encrypting images due to their high computational complexity and some other constrains. The algorithm of the chaos-based image cryptosystem is depicted in Fig. 4. We consider a generalized projective series of four chaotic systems, so that we can use the variables of any system as the secret keys for the encryption process. The standard procedure of diffusion and confusion can be performed to encrypt the image and will send it to the public channel. The receiver is also a generalized projective series of synchronized chaotic 
systems, successfully generates the secret keys from their own dynamics and using the inverse process successfully gets back to the original image after decryption.

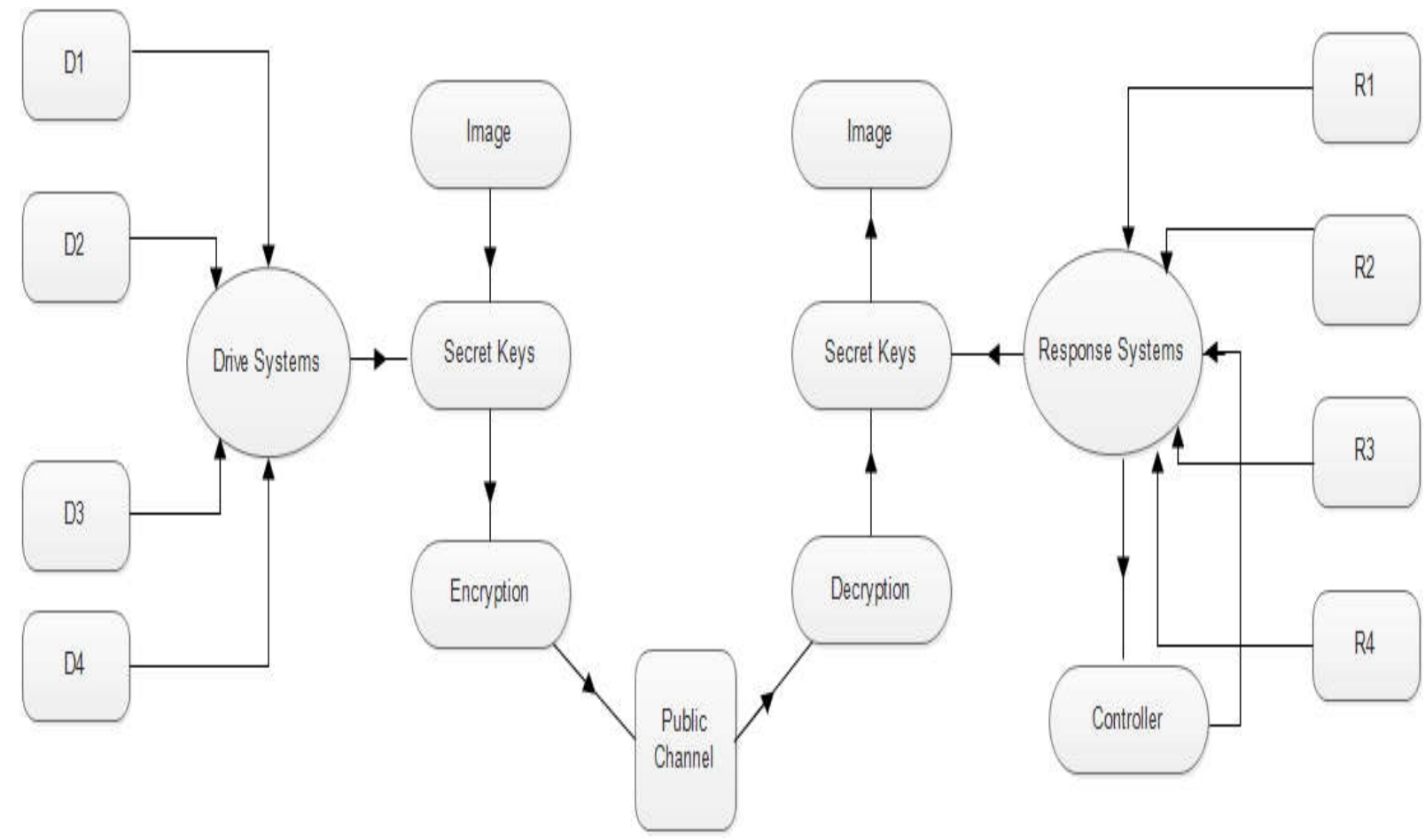

Fig.4.Image encryption algorithm

Pixel position is scrambled over the entire image without disturbing the value of the pixels and the image becomes unrecognizable. The initial conditions and control parameters of any systems (1)-(4) are used as the secret keys. Here, we use different chaotic systems to have better security. To enhance the security, the second stage of encryption is carried out aiming at changing the value of each pixel in the whole image. This diffusion stage is accomplished by any chaotic systems (1)-(4), where the initial conditions and its control parameters are used as the secret keys.

Reversely for decryption process, first stage is the diffusion stage. The chaotic systems (1)-(4) is used to retrieve pixels' original values. Confusion stage is carried out for the next stage of decryption process. The permutation of pixel position is implemented by using chaotic systems (1)-(4). The same initial conditions and control parameters for generating the chaos sequence were used in encryption and decryption process for both confusion and diffusion stages. Therefore, in decryption process, the same chaotic systems with the same diffusion key are used to get the original pixel values while the same chaotic systems with the same 
confusion key are used to get the original position of the image. The output of decryption system gives the original image.

\subsection{A Simple Example}

The original image taken for the work is given in Fig 5(a). The encrypted image after the procedure of confusion and diffusion take part is shown in Fig. 5(b). We also analyzed the correlation between adjacent pixels in original image and encrypted image. The procedure is as follows:

$r_{x, y}=\frac{\operatorname{cov}(x, y)}{\sqrt{D(x)} \sqrt{D(y)}}(12)$

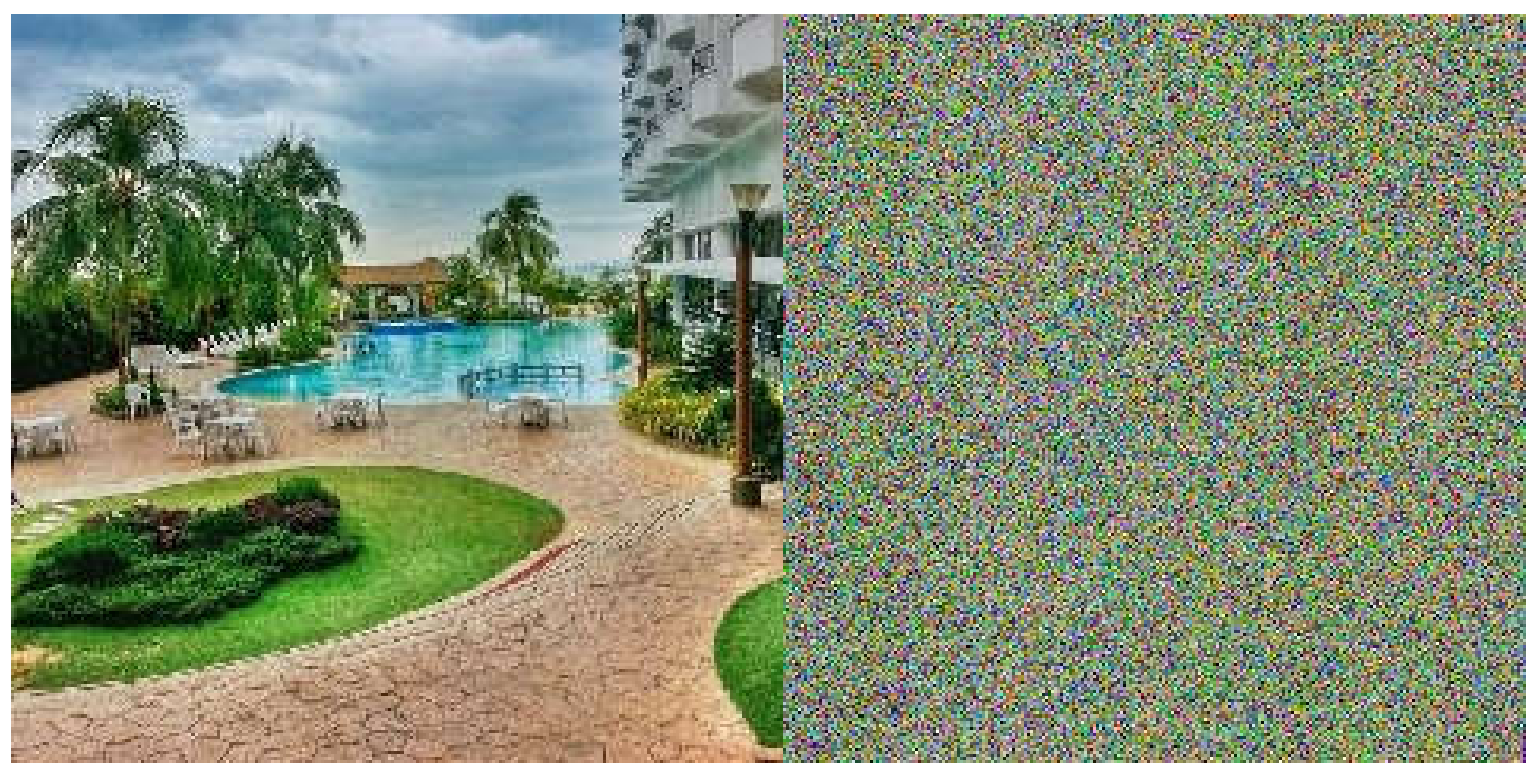

(a)

(b)

Fig.5(a) The original image, (b) The encrypted image

where $x$ and $y$ are the values of two adjacent pixels in the image. Fig. 6(a) is the correlation coefficient of the original image, while the Fig. 6(b) is the correlation coefficient of the encrypted image. 


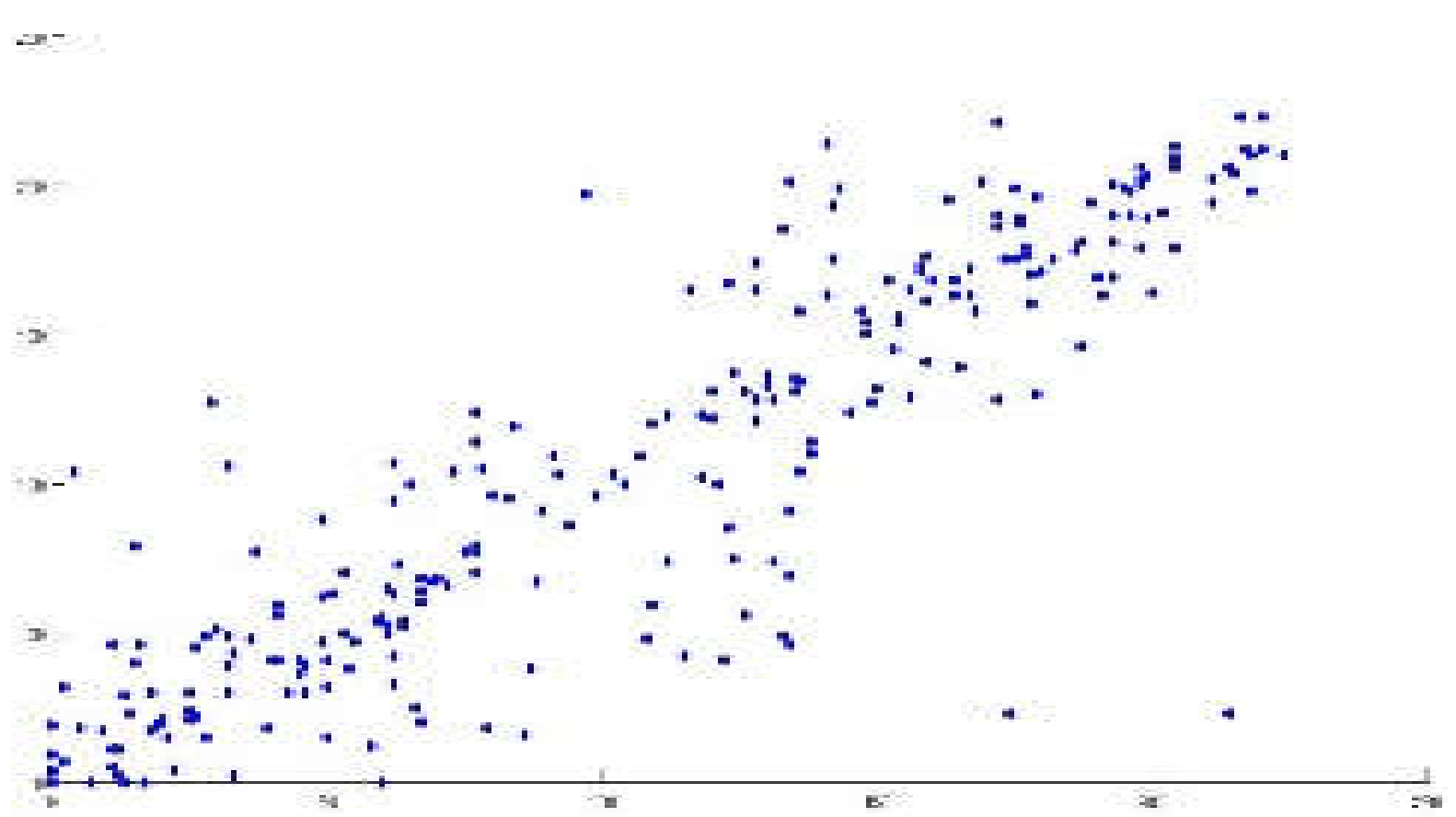

(a)

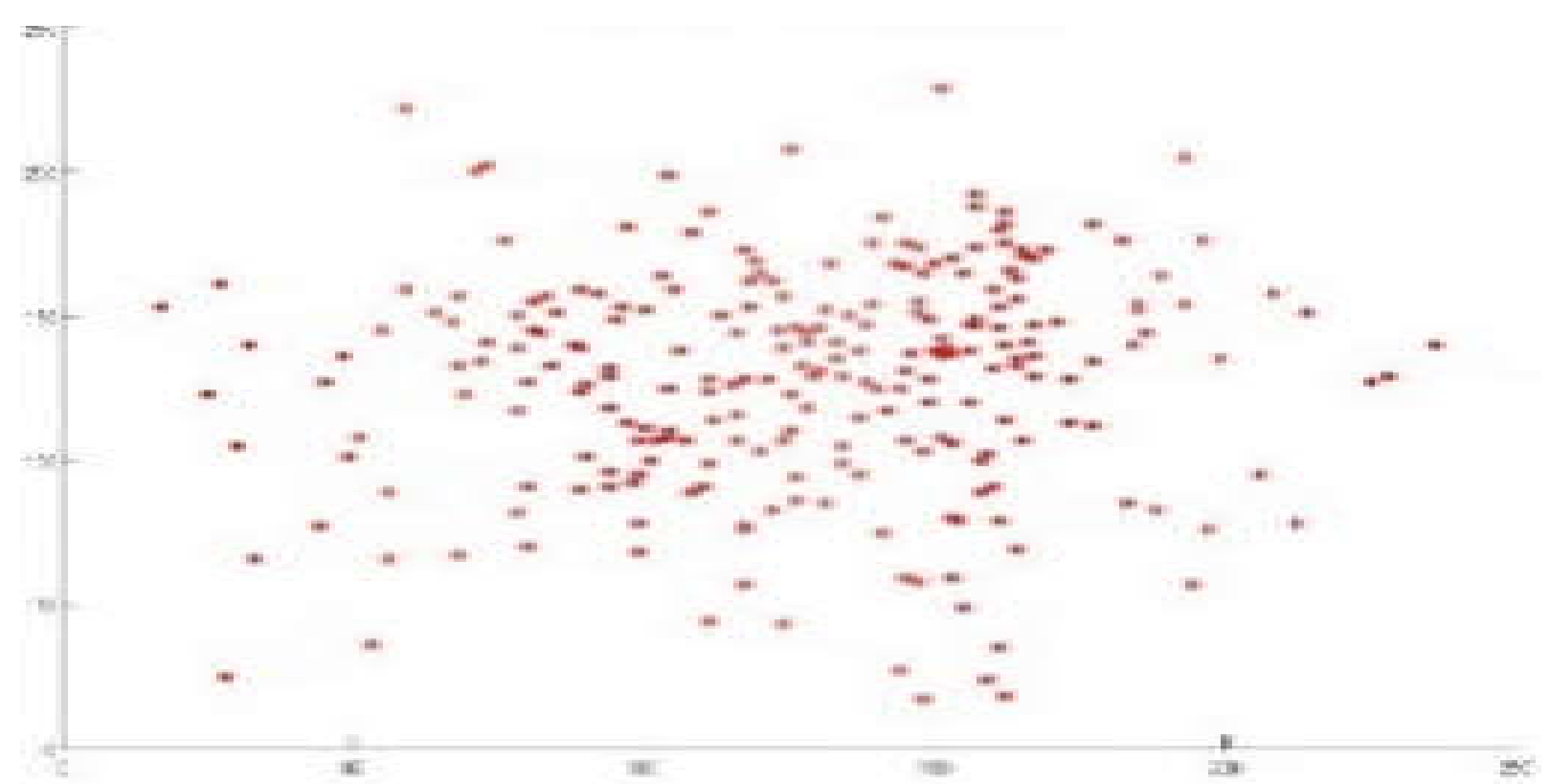

(b)

Fig.6(a). The correlation coefficient of the original image, (b)The correlation coefficient of the encrypted image

\section{CONCLUSION}

We investigate the generalized projective series synchronization between four different chaotic systems by using the technique of active control. The designed active controller ensures that stabilization of synchronization between drive and response systems. Numerical 
simulation was also important to illustrate the effectiveness of the approach. The sufficient conditions for synchronization is also derived. This kind of phenomenon can be well implemented in the image encryption and decryption algorithm. A new image encryption scheme was designed. The proposed algorithm shuffles the positions and changes the values of image pixels simultaneously with the help of sequence generated by four different chaotic systems. This algorithm can be carried out for encrypting gray-scale image as well as true colour image. In conclusion, the proposed algorithm is secure and practical.

\section{ACKNOWLEDGEMENTS}

The author would like to thank the reviewers for their constructive suggestions. This paper was funded by National Defence University of Malaysia.

\section{REFERENCES}

[1] Dashselt F, Schwatrz W. Chaos and cryptography. IEEE Transactions on Circuits and Systems I: Fundamental Theory and Applications, 2011, 48(12):1498-1509

[2] Renato E, Steven H. Synchronization of pulse-coupled biological oscillators. SIAM Journal on Applied Mathematics, 1989, 50(60):1645-1662

[3] Parmananda P, Jiang Y. Synchronization of chemical systems using external forcing. Journal of Physical Chemistry A., 2012, 102(24):4532-4536

[4] Minghui X, Tieri D, Fine E, Thompson J, Holland M. Synchronization of two ensembles of atoms. Physical Review Letters, 2014, 113(15):1-5

[5] Bloch G, Herzog E, Levine J, Schwartz W. Socially synchronized circadian oscillators. Proceedings of the Royal Society of London B: Biological Sciences, 2013, 280(1765):1-10 [6]Banerjee S, Ghosh D, Chowdhury A. Multiplexing synchronization and its applications in cryptography. PhysicaScripta, 2008, 78(1):1-7

[7] Banerjee, S Saha P, Chowdhury A. Some aspects of synchronization and chaos in a coupled laser system. Chaos, Solitons and Fractals, 2002, 14(7):1083-1093

[8] Jovic B. Synchronization techniques for chaotic communication systems.Berlin: Springer, 2011 
[9] Zhan M, Wei W G, Lai C H. Transition from intermittency to periodicity in lag synchronization in coupled Rossler oscillators. Physical Review E, 2002, 65(3):1-5

[10] Ma J, Li F, Huang L, Jin W Y. Complete synchronization, phase synchronization and parameters estimation in a realistic chaotic system. Communications in Nonlinear Science and Numerical Simulation, 2011, 16(9):3770-3785

[11] Ji D H, Jeong S C, Park J H, Lee S M, Won S C. Adaptive lag synchronization for uncertain complex dynamical network with delayed coupling. Applied Mathematics and Computation, 2012, 218(9):4872-4880

[12] Boccaletti S, Kurths J, Osipov G, Valladares D, Zhou C. The synchronization of chaotic systems. International Journal of Advances Information Technology, 2002, 366(1):1-101

[13] Zan L K, En H, Rong Z Z, Tse C K. Generalized projective synchronization of two coupled complex networks of different sizes. Chinese Physics B, 2013, 22(7):1-7

[14] Wang S, Yu Y, Wen G. Hybrid projective synchronization of time-delayed fractional order chaotic systems. Nonlinear Analysis: Hybrid Systems, 2014, 11:129-138

[15] Sawalha M. Hybrid adaptive synchronization of hyperchaotic systems with fully unknown parameters. Applied Mathematics, 2013, 4(12):1621-1628

[16] Zhan M, Wang X, Gong X, Wei G W, Lai C H. Complete synchronization \& generalized synchronization of 1 way coupled time delay systems. Physical Review E, 2003, 68(3):1-5

[17] Sawalha M. On projective synchronization of hyperchaotic complex nonlinear systems based on passive theory for secure communications. PhysicaScripta, 2013, 87(5):1-10

[18] Boulkroune A, Bouzeriba A, Bouden T. Fuzzy generalized projective synchronization of incommensurate fractional-order chaotic systems. Neurocomputing, 2016, 173:606-614

[19] Ping L, Shutang L. Anti-synchronization between different chaotic complex systems.PhysicaScripta, 2011, 83(6):1-9

[20] Boccaletti S, Farini A, Arecchi F. Adaptive synchronization of chaos for secure communication. Physical Review E, 1997, 55(5):4979-4981

[21] KuetcheMbe E S, Fotsin H B, Kengne J Woafo P. Parameters estimation based adaptive Generalized Projective Synchronization (GPS) of chaotic Chua's circuit with application to chaos communication by parametric modulation. Chaos, Solitons and Fractals, 2014, 


\section{1:27-37}

[22] Tianfang C, Tianxing C, Zhou C, Yu M. Encryption using two non-identical chaotic systems. PhysicaScripta, 2002, 66(2):187-192

[23] Banerjee S, Mukhopadhyay S, Rondoni L. Multi-image encryption based on synchronization of chaotic lasers and iris authentication. Optics and Laser in Engineering, 2012, 50(7):950-957

[24] Mondal B, Mondal T. A Nobel chaos based secure image encryption algorithm. International Journal of Applied Engineering Research, 2016, 11(5):3120-3127

[25] Yoshimura K. Multichannel digital communication by the synchronization of globally coupled chaotic systems. Physical Review E, 1999, 60(2):1648-1657

[26] Liu Y, Davis P. Dual synchronization of chaos. Physical Review E, 2000, 61(3):2176-2179

[27] Ning D, Lu J, Han X. Dual synchronization based on two different chaotic systems: Lorenz systems and Rossler systems. Journal of Computational and Applied Mathematics, 2007, 206(2):1046-1050

[28] Junwei S, Suxia J, Guangzhao C, Yanfeng W. Dual combination synchronization of six chaotic systems. Journal of Computational and Nonlinear Dynamics, 2016, 11(3):1-5

[29] Othman A A, Noorani M S M, Al-Sawalha M M. Dual synchronization of chaotic and hyperchaotic systems. Journal of Nonlinear Sciences andApplications, 2016, 9(6):4666-4677

[30] Sprott J. Some simple chaotic flows. Physical Review E., 1994, 50(2):647-650

[31] Mukherjee P, Banerjee S. Projective and hybrid projective synchronization for the Lorenz-Stenflo system with estimation of unknown parameters. PhysicaScripta, 2010, $82(5): 1-5$

[32] Stenflo L. Generalized Lorenz equation for acoustic-gravity waves in the atmosphere. PhysicaScripta, 1996, 53(1):83-84

\section{How to cite this article:}

Fataf N A A, Said M R M. Generalized projective series synchronization between chaotic systems and its application. J. Fundam. Appl. Sci., 2017, 9(3S), 294-307. 